\title{
Significance of arterial spin labeling perfusion and susceptibility weighted imaging changes in patients with transient ischemic attack: a prospective cohort study
}

Inger Havsteen ${ }^{1 *} \mathbb{D}$, Lasse Willer ${ }^{2}$, Christian Ovesen², Janus Damm Nybing ${ }^{1}$, Karen Ægidius ${ }^{2}$, Jacob Marstrand ${ }^{2}$, Per Meden ${ }^{2}$, Sverre Rosenbaum², Marie Norsker Folke ${ }^{2}$, Hanne Christensen ${ }^{2}$ and Anders Christensen ${ }^{1}$

\begin{abstract}
Background: In a prospective cohort of patients with transient ischemic attack (TIA), we investigated usefulness and feasibility of arterial spin labeling (ASL) perfusion and susceptibility weighted imaging (SWI) alone and in combination with standard diffusion weighted (DWI) imaging in subacute diagnostic work-up. We investigated rates of ASL and SWI changes and their potential correlation to lasting infarction 8 weeks after ictus.

Methods: Patients with TIA underwent 3T-MRI including DWI, ASL and SWI within $72 \mathrm{~h}$ of symptom onset. We defined lasting infarction as presence of 8-week MRI T2-fluid attenuated inversion recovery (FLAIR) hyperintensity or atrophy in the area of initial DWI-lesion.

Results: We included 116 patients. Diffusion and perfusion together identified more patients with ischemia than either alone ( $59 \%$ vs. $40 \%, p<0.0001)$. The presence of both diffusion and perfusion lesions had the highest rate of 8 -week gliosis scars, $65 \%(p<0.0001)$. In white matter, DWI-restriction was the determinant factor for scar development. However, in cortical gray matter half of lesions with perfusion deficit left a scar, while lesions without perfusion change rarely resulted in scars (56\% versus $21 \%, p=0.03)$. SWI lesions were rare $(6 \%)$ and a subset of perfusion lesions. SWI-lesions with DWI-lesions were all located in cortical gray matter and showed high scar rate.
\end{abstract}

Conclusions: ASL perfusion increased ischemia detection in patients with TIA, and was most useful in conjunction with DWI. ASL was fast, robust and useful in a subacute clinical diagnostic setting. SWI had few positive findings and did not add information.

Trial Registration.: http://www.clinicaltrials.gov. Unique Identifier NCT01531946, prospectively registered February 9, 2012.

Keywords: Transient ischemic attack, Cerebral cortex, Arterial spin labeling

\section{Background}

Diagnosing a patient with transient ischemic attack (TIA) clinically and evaluating the stroke risk hereafter [1] is a challenge even to experienced stroke neurologists [2]. The addition of noninvasive arterial spin labeling (ASL) perfusion imaging to standard TIA-MRI protocol has been shown to significantly increase the MRI

\footnotetext{
* Correspondence: Inger.birgitte.havsteen@regionh.dk

'Department of Radiology, Copenhagen University Hospital Bispebjerg,

Bispebjerg Bakke 23, 2400 Copenhagen, NV, Denmark

Full list of author information is available at the end of the article
}

detection [3] of ischemic findings. Increased reliability, [4] accessibility and automatization of post-processing have turned ASL perfusion into a clinically applicable tool [5] in stroke [6-9] and TIA [3, 10-12]. Focal ASL changes in cerebral blood flow (CBF), $[13,14]$ and arterial transit time, $[12,15,16]$ are well described in TIA populations. $[3,10,14]$ Based on ASL, lesions are estimated to occur in $35-56 \%[3,17]$ of patients with TIA. In patients with no diffusion lesions, contrast-enhanced dynamic susceptibility contrast (DSC) perfusion imaging detects abnormalities in $16-32 \%[18,19]$ and in $18-46 \%[3,17]$ with both

(c) The Author(s). 2018 Open Access This article is distributed under the terms of the Creative Commons Attribution 4.0 International License (http://creativecommons.org/licenses/by/4.0/), which permits unrestricted use, distribution, and 
DSC and ASL. Also, perfusion changes in diffusion negative patients with clinical TIA are associated with higher rates of new diffusion weighted imaging (DWI) lesions on 3 -day follow-up [20, 21]. It is conceivable that originally hypoperfused areas may progress to critical ischemia indicated by new DWI or T2-FLAIR lesions on follow-up [11, 19]. In addition, perfusion changes might also hold predictive power towards risk for de-novo future ischemic events. Consequently, the usefulness of ASL as standard add-on sequence to DWI in a subacute clinical setting without off-line post-processing is an open question.

Findings of asymmetric veins based on $\mathrm{T}_{2}^{*}$ and susceptibility weighted imaging (SWI) have also been described in stroke populations and correspond approximately to DSC hypoperfusion areas [22-25] and infarct volume at 72-h follow-up [26]. To our knowledge, SWI and findings in TIA are not described in literature. As the ischemic event in TIA is transitory and presumably less profound than in stroke, SWI may supplement ASL, reflecting venous output from ischemic tissues and indicating the depth of perfusion disturbance.

Optimizing the diagnostic yield of the various sequences included in the TIA work-up protocol is important, as since prolonged scan time decreases the quality of diagnostic evaluation due to the increased incidence of motion artefacts. Motion artefacts increase with increasing scan time, $[27,28]$ presence of pathology [29] and in acute settings [30].

Consequently, we aimed to investigate if standard DWI plus ASL and SWI compared to DWI alone increases the neuroradiological detection of ischemia in patients with TIA in a subacute clinical setting. In addition, we tested if ASL and SWI added to the prediction of 8-week infarction signs and recurrent cerebrovascular events after TIA.

\section{Methods}

We studied a prospective cohort of patients with TIA admitted February 2012 - December 2014 to our comprehensive stroke center. Patients were included after own written informed consent within $72 \mathrm{~h}$ of symptom onset. The study was approved by the National Committee of Biomedical Research Ethics (H-1-2011-75, ClinicalTrials.gov Identifier NCT01531946).

\section{Clinical assessment}

Senior consultant stroke neurologists clinically evaluated patients. Inclusion criteria were admission with acute focal neurological symptoms believed to be of vascular origin. Exclusion criteria were MRI contraindications, non-TIA discharge diagnosis, and severe comorbidity. We defined TIA as acutely appearing focal neurological deficit with resolution within $24 \mathrm{~h}$. Resolution was defined as National Institute of Health Stroke Scale (NIHSS) 0.
Patients were treated in accordance with European and national guidelines. In this study an ischemic event was defined based on either radiological signs of ischemia or clinical judgment in imaging negative patients.

Recurrent ischemic events were defined as new TIA or stroke diagnosis with clear temporal separation from the index event. We performed long-term follow-up by national electronic patient files.

\section{Study procedures}

All patients included in this study underwent the baseline sub-acute MRI during their admission at the stroke unit. The second MRI was obtained after 8 weeks as an outpatient procedure.

\section{Imaging}

Imaging was performed at 3T (Siemens Magnetom Verio, Siemens, Erlangen, Germany) with a 32-channel head coil (Siemens, Erlangen, Germany). The baseline imaging protocol consisted of ASL, SWI, and DWI and T2-FLAIR used in our routine TIA protocol. At 8-week follow-up MRI, we scanned only DWI and T2-FLAIR.

We used Siemens' 3D background suppressed ep2d pulsed ASL sequence, TR $2500 \mathrm{~ms}$, TE $11 \mathrm{~ms}, 192-\mathrm{mm}$ FOV, FA 90, voxel size $3 \times 3 \times 6 \mathrm{~mm}^{3}$, TI1 $=700 \mathrm{~ms}$, TI2 $=$ $1800 \mathrm{~ms}$, acceleration factor $R=2$, acquisition time 4:22 min:s. Perfusion weighted images, motion corrected relative $\mathrm{CBF}$ and the intrinsic motion correction series were sent to PACS. The SWI protocol was TR $27 \mathrm{~ms}$, TE $20 \mathrm{~ms}, 240$-mm FOV, FA 15, matrix $243 \times 256,1.0 \times 0.9 \times$ $1.5 \mathrm{~mm}^{3}$ voxel size, 80 slices, acquisition time 5:18 min:s. ASL and SWI-postprocessing were fully automated as provided by the vendor.

The DWI was single-shot spin-echo diffusion echoplanar imaging with 220-mm FOV, 25 4-mm axial slices with 0 -mm gap, b-value $0,1000 \mathrm{~s} / \mathrm{mm}^{2}$ along 3 orthogonal axes; TR $6600 \mathrm{~ms}$, TE $100 \mathrm{~ms}$, acceleration factor $\mathrm{R}=2$, matrix $192 \times 192$. ADC maps were automatically generated as provided by the vendor.

For T2-FLAIR we used 240-mm FOV, 27 4-mm axial slices with 0-mm gap, TR $6500 \mathrm{~ms}$, TE $133 \mathrm{~ms}$, TI $2134 \mathrm{~ms}$, acceleration factor $\mathrm{R}=2$, matrix $256 \times 256$.

\section{Image analysis}

In this study we used only the PACS and no external software. Between sequences and MRIs performed at baseline and 8-weeks we identified lesions through their location, describing their position with two orthogonal intrathecal diameters. To provide a standardized description we developed a case report form (CRF) and literature-based image template with defined scoring categories. We validated CRF and template on 50 randomly chosen cases from the cohort with two blinded board-certified consultant neuroradiologists $(\mathrm{AC}, \mathrm{IH})$ reading first 
independently and then jointly establishing final consensus on the reading tools. Hereafter one reader $(\mathrm{IH})$, blinded to clinical information except for the referral information, systematically assessed all baseline and 8-week imaging sequences in accordance with the predefined CRF for ASL perfusion changes, DWI-lesions, SWI asymmetric veins and missing flow voids and lesion visibility on T2-FLAIR (Fig. 1). We scored ASL, SWI and DWI images separately to avoid bias. We categorized lesion location according to vascular territory and as hemispheric left-, right, bilateral or infratentorial. For quality control we calculated intraobserver variability for two CRF readings, except for area measurements, at 3 months' interval in a 10\% sample (patients born 4th, 14th and 24th day in any month) and found substantial agreement (Cohen's kappa $=0.80$ ).

As motion artefacts may compromise ASL interpretation, we defined the threshold for permissible motion as motion in one plane, usually physiological motion in cranio-caudal direction, and motion presence in less than $2 / 3$ of the whole brain series' images. ASL image quality assessment is detailed in Additional files 1 and 2.

The arterial transit artefact (ATA) depicts areas where CBF may be normal, but where arterial transit time is increased, and is seen as vivid serpiginous high signal of labeled blood stagnated in precapillary cortical vessels $[15,16,31]$. We deemed ASL positive, when at least one of the following was present: focal low CBF, ATA or focal hyperperfusion (Fig. 1). SWI asymmetric prominent veins were defined as regions of multiple hypointense vessels [25] and dichotomized into presence and absence. We accepted newfound focal gliosis or atrophy on 8-week follow-up MRI as marker for scar tissue after neuronal death and thus critical ischemic depth [32].

We investigated lesions' tissue localization stratifying lesions after their DWI location into white matter (WM), cortical gray matter (cGM) and deep gray matter (dGM).

After finalized clinical and radiological data collection, radiological lesion localization was compared with clinical symptoms for consistency under supervision of a senior neurological consultant (HC).

\section{Statistical analysis}

Categorical data were analyzed using Fisher's exact test. Differences in continuous and ordinal data were analyzed using Mann-Whitney U test, and for differences between proportions we used McNemar-test. Linear relations between variables and a dichotomous outcome were tested using bivariate logistic regression. Un-adjusted survival analysis for recurrent cerebrovascular event was conducted using Kaplan-Meier curves and log-rank test. Relation between imaging findings and recurrent cerebrovascular event was analyzed using Cox Proportional Hazard Model adjusting for other risk factors. Data were presented as frequencies, medians with interquartile range (IQR), odds ratios (OR) or hazard ratios (HR) with 95\%

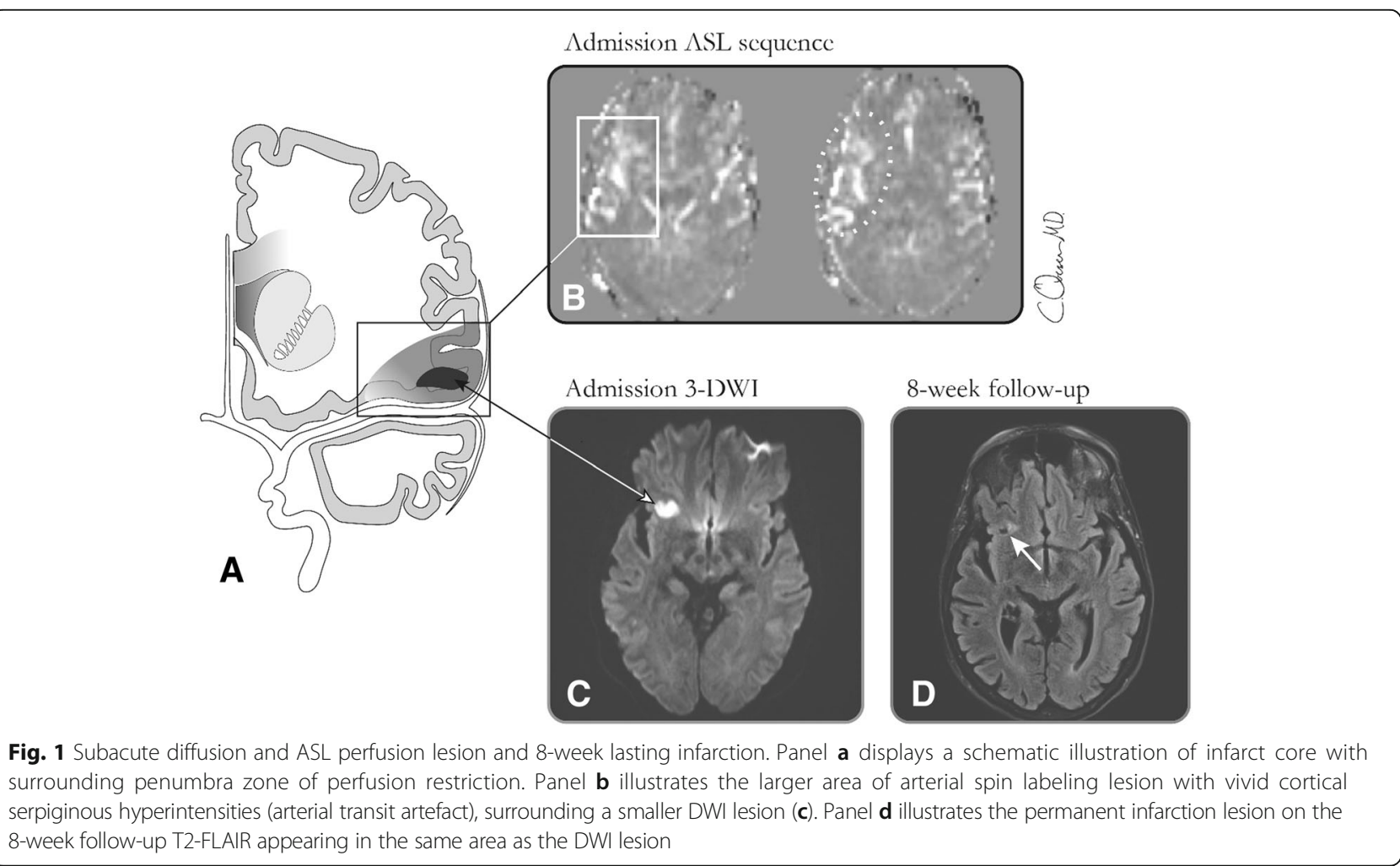


confidence intervals $(\mathrm{CI})$ as appropriate. $P$-values less than 0.05 were considered significant. Statistical analysis was performed with RStudio (Version 0.97.168) 2012 RStudio Inc., Boston, MA, USA and SPSS (version 20) statistical software (IBM Corp, Armonk, New York, USA).

\section{Results}

\section{Population}

Patient flow is described in (Fig. 2). Median (IQR) age was 65 (54-71) years, 43\% were female, and median (IQR) ABCD2 was 4 (3-5). Table 1 shows detailed patient characteristics. The median (IQR) time from ictus to initial MRI was 38 (24-58) hours. Scans from 4 patients had unacceptable motion artifacts, and 2 patients had no ASL done due to a technical error, these were excluded from the analysis. ASL and SWI with 8-week follow-up MRI were consequently available for 116 patients with 148 clinical or radiological events, hereof were 100 visible ischemic lesions on at least one sequence. We differentiated between patients and lesions, as patients may have multiple lesions in several vascular territories and not all foci showed perfusion changes.

Among patients $46(40 \%)$ had at least one DWI lesion and $46(40 \%)$ showed ASL abnormalities, 24 patients were both DWI and ASL positive (Table 1). Including both DWI and ASL as diagnostic markers of ischemia increased ischemia detection from $46(40 \%)$ to 68 (59\%) of 116 patients $(p<0.0001)$. In the cohort 148 acute clinical or radiological events were detected. Of these, 79 (53\%) were DWI positive and $68(46 \%)$ were ASL positive lesions. Diffusion and perfusion were both positive in $47(32 \%)$ lesions and 21 lesions (14\%) were ASL-

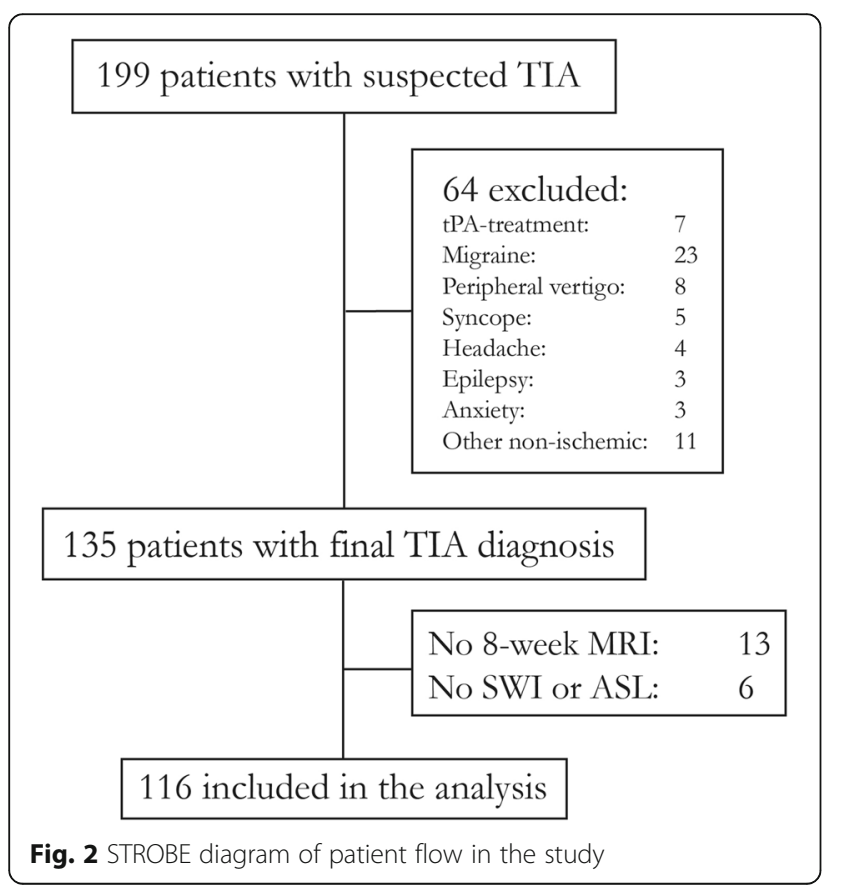

Table 1 Patient characteristics

\begin{tabular}{|c|c|}
\hline All patients & 116 \\
\hline Female sex & $50(43 \%)$ \\
\hline Age, median (IQR) & $65(54-71)$ \\
\hline \multicolumn{2}{|l|}{ Medical history: } \\
\hline Prior stroke & $21(17 \%)$ \\
\hline Prior TIA & $10(9 \%)$ \\
\hline Prior Ml & $9(8 \%)$ \\
\hline Atrial fibrillation & $12(10 \%)$ \\
\hline Hypertension & $57(49 \%)$ \\
\hline Diabetes & $15(13 \%)$ \\
\hline Depression & $11(10 \%)$ \\
\hline Current smoking & $40(35 \%)$ \\
\hline Alcohol overuse & $10(9 \%)$ \\
\hline Antiplatelet use & $38(33 \%)$ \\
\hline Warfarin use & $0(0 \%)$ \\
\hline \multicolumn{2}{|l|}{ Index stroke: } \\
\hline ABCD2, median (IQR) & $4(3-5)$ \\
\hline \multicolumn{2}{|l|}{ Duration of symptoms: } \\
\hline$<60 \min$ & $56(48 \%)$ \\
\hline$>60 \mathrm{~min}$ & $60(52 \%)$ \\
\hline \multicolumn{2}{|l|}{ TOAST etiology: } \\
\hline Small vessels & 47 (41\%) \\
\hline Large vessels & $26(22 \%)$ \\
\hline Cardiogenic & $18(16 \%)$ \\
\hline Multiple possible etiologies & $25(22 \%)$ \\
\hline TTS, median (IQR) & $38(24-58)$ \\
\hline TTF, median (IQR) & $56(55-60)$ \\
\hline \multicolumn{2}{|l|}{ Radiological findings: } \\
\hline DWI positive patients & $46(40 \%)$ \\
\hline Lesions, n. & 79 \\
\hline ASL positive patients & $46(40 \%)$ \\
\hline Hypoperfusion lesions, n. & 38 \\
\hline Hyperperfusion lesions, n. & 22 \\
\hline ATA, $n$. & 28 \\
\hline SWI positive patients & $5(4 \%)$ \\
\hline SWI lesions, n. & 6 \\
\hline
\end{tabular}

Numbers are frequency (\%) unless otherwise indicated. IQR interquartile range, TIA transient ischemic attack, MI myocardial infarction, NA not applicable, TTS time to scan, TTF time to follow-up, ATA arterial transit artefact

positive and DWI-negative. Diffusion and ASL findings in patients are detailed in Fig. 3. Among the 68 perfusion lesions in 46 patients, ATA was present in 24 $(52 \%, p<0.0001)$ patients with perfusion changes and in relation to $20(53 \%, p<0.0001)$ hypoperfusion lesions. One patient with several lesions showed foci with hypoperfusion-ATA combination and foci with 
Total number of patients, $\mathrm{n}=116$

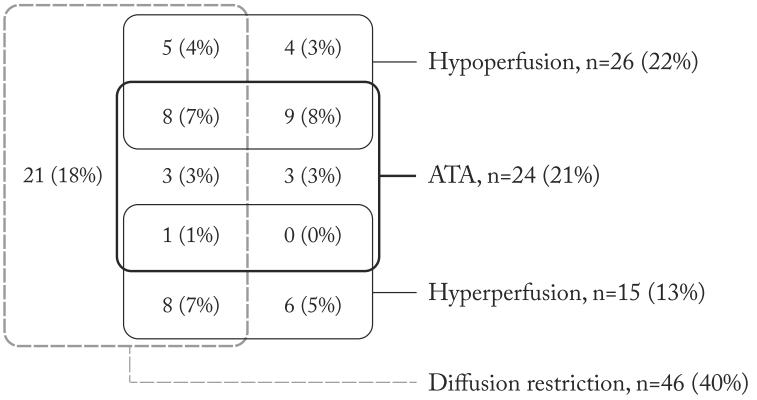

Diffusion and perfusion negative patients, $\mathrm{n}=48$ (41\%)

Fig. 3 Wenn diagram of diffusion and perfusion findings in patients

hyperperfusion (Fig. 3). There were no distinct lesions with discernible combinations of ATA and hyperperfusion or of hypo- and hyperperfusion.

\section{Characteristics of patients with 8-week infarction signs}

In patients with DWI-lesions, a subsequent scarring was observed in $85 \%$ at 8 weeks. There was a significant difference between the different TOAST categories and the probability of ASL-lesion with small vessel etiology yielding the lowest probability of ASL-lesion (19\%), and large vessel etiology yielding the highest probability $(65 \%, p<0.001)$. Adjusted for the presence of a DWI lesion, large vessel (OR 8.08, 95\% CI 2.66-24.52) and multiple possible etiologies (OR 5.27, 95\% CI 1.76-15.75) yielded a higher probability of ASL-lesion than either small vessel etiology (reference category) or cardiogenic etiology (OR 1.97, 95\% CI 0.57-6.84). Detailed large vessel findings are shown in Additional file 1: Table S2. Adding ASL to initial DWI does not increase diagnostic accuracy of permanent infarction development (Table 2).

During the median (IQR) follow-up period of 1237 (771-1536) days, 17 patients suffered a recurrent

Table 2 Diagnostic accuracies of DWI and ASL findings for 8-week infarction in patients and lesions

\begin{tabular}{llllll}
\hline Patients & sensitivity & specificity & PPV & NPV & accuracy \\
\hline DWI+ & 0.98 & 0.91 & 0.85 & 0.99 & 0.93 \\
ASL+ & 0.58 & 0.68 & 0.49 & 0.75 & 0.65 \\
DWI+, ASL+ & 0.98 & 0.62 & 0.57 & 0.98 & 0.74 \\
DWI+, ASL- & 0.40 & 0.93 & 0.76 & 0.75 & 0.75 \\
DWI-, ASL+ & 0 & 0.71 & 0 & 0.57 & 0.47 \\
Lesions & & & & & \\
DWI+ & 0.98 & 0.71 & 0.65 & 0.99 & 0.80 \\
ASL+ & 0.60 & 0.61 & 0.46 & 0.61 & 0.61 \\
DWI+, ASL+ & 0.60 & 0.83 & 0.66 & 0.79 & 0.75 \\
DWI+, ASL- & 0.38 & 0.88 & 0.63 & 0.72 & 0.72 \\
DWI-, ASL+ & 0 & 0.78 & 0 & 0.59 & 0.51 \\
\hline
\end{tabular}

cerebrovascular event. Patients with initial ASL lesion (Fig. 4) did not show a higher risk of recurrent cerebrovascular event (HR 1.87, 95\% CI 0.70-4.97) after adjusting for presence of DWI-lesion and ABCD2.

\section{Characteristics of lesions with 8-week infarction signs}

A total of $65 \%$ of DWI-lesions and $45 \%$ of ASL-lesions subsequently developed a scar on 8-week MRI. We found the scar-rate to be highest in lesions showing both diffusion restriction and altered perfusion (65\%), however only slightly, and non-significantly higher than DWI positive and ASL negative patients (64\%). The type of perfusion finding, e.g. ATA, showed no effect on the odds for persistent infarction sign development (Additional file 1: Table S3).

In WM DWI lesions had a high probability of scarring independent of the presence of perfusion abnormalities (94\% vs. 92\%, $p=0.765$ ). In cGM DWI lesions were significantly more likely to develop subsequent infarction after 8 weeks if the lesions was ASL positive (56\% vs. $21 \%, p=0.029$ ). The median (IQR) size of the DWI lesions located in the cGM was not significantly larger than those located in the WM (28.0 (14.0-51.0) vs. 24.5 $\left.(9.0-59.0) \mathrm{mm}^{2} ; p=0.463\right)$. There was a significant correlation between the size of the DWI lesion and probability of surrounding ASL lesion (OR 1.02, 95\% CI 1.01-1.03 per additional $\mathrm{mm}^{2}$ ).

We had too few $(n=4)$ deep gray matter lesions to conduct a meaningful analysis.

\section{SWI findings}

As a subset of patients with ASL abnormalities, we found 5 patients (with 6 lesions) with SWI abnormalities. In only one patient no corresponding DWI lesion was found to the ASL and SWI abnormality; this lesion did not show any scarring at the 8-week MRI. All 5 diffusion positive lesions were located in the cortical gray matter, and 4 (80\%) lesions were followed by 8 -week gliosis scars; this scar rate was higher than the $52 \%$ scar-rate in patients with cGM lesions with ASL abnormalities without SWI findings, though not statistically significant $(p=0.355)$. SWI abnormalities occurred both in focal hypoperfusion (2/6), ATA (2/6) and hyperperfusion $(3 / 6)$.

\section{Discussion}

This study showed that using both diffusion and ASL perfusion imaging identified significantly more patients as MRI-imaging positive for acute vascular findings than DWI alone in a subacute clinical setting without external software. DWI-lesion presence seemed to be the decisive factor for determining lasting infarct changes after TIA, especially in white matter. DWI lesions in cortical gray matter without perfusion change developed few scars, 

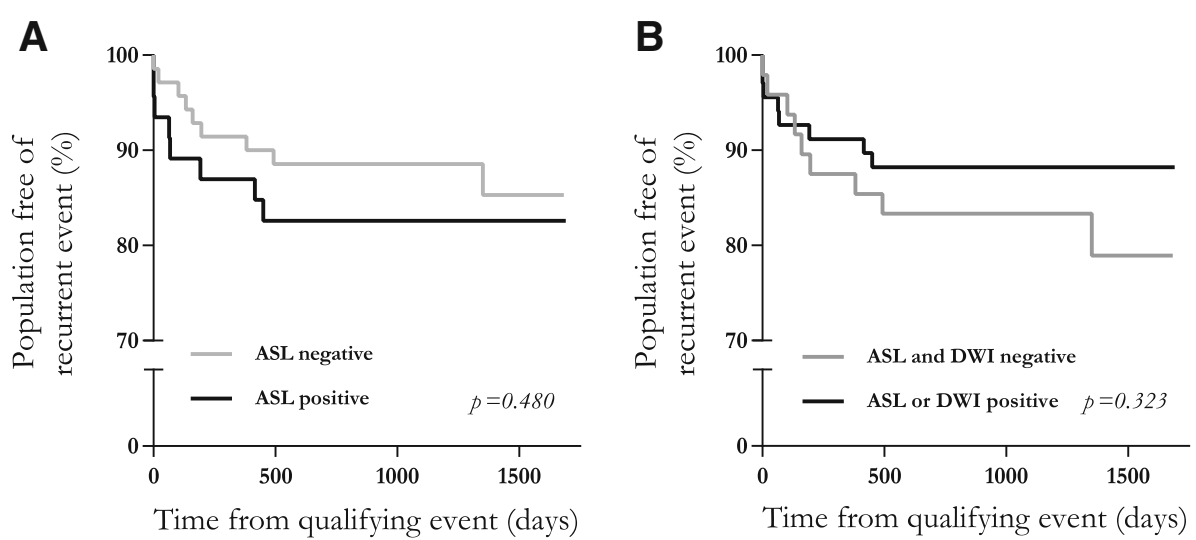

Fig. 4 Event recurrence stratified by lesion characteristics. Two Kaplan-Meier curves showing recurrent cerebrovascular event in the TIA population stratified by MRI-sequence positivity. Panel a shows that patients with ASL perfusion lesions do not experience a higher risk of recurrent cerebrovascular events compared to TIA patients displaying no perfusion lesion. Panel $\mathbf{b}$ : shows that radiological evidence of acute ischemia (either restricted perfusion or diffusion) does not identify patients in higher risk of recurrent event

while half of lesions with perfusion change left a scar. SWI did not add to detection of ischemia in TIA, but may indicate profound perfusion disturbance.

Our study has a high frequency of lesions, most likely because of inclusion of well-defined patients and subsequent exclusion of patients with other final diagnoses than TIA. This hampers generalizability to less selected emergency room patients concerning lesion frequency, however not regarding the natural MRI history of ischemic lesions after TIA. The rates of DWI lesions are reported to vary between 25 and $50 \%[3,17,18,33-36]$ and to be halved in populations with high stroke awareness and easy-access high-volume TIA clinics [37].

Other potential limitations are: Motion artifacts increase with scan time $[27,28,38]$ and may compromise perfusion parameter estimates [5]. We had too few deep gray matter lesions and SWI positives for meaningful subgroup analysis. Our SWI positive lesions were a subset of perfusion positives that with concomitant DWI lesions showed high scarring rate. This may indicate that visible venous congestion serves as marker for focal ischemic depth, exploration would need more data.

We confirmed that the combination of diffusion and ASL perfusion identifies significantly more lesions than either sequence alone in TIA, $[3,14]$ from $40 \%$ up to nearly $60 \%$ of TIA patients in our subacute clinical setting. Roughly half of white matter lesions showed perfusion change compared to three quarters of cortical lesions: This presumably reflects the local vascularity with rich collaterals cortically and fewer in deeper tissue, relying on perforants, [39-41] and the longer and more heterogeneous transit times of white matter [42, 43] decreasing ASL's sensitivity to focal changes. This also explains the lower probability of ASL changes in patients with small vessel etiology. Larger cortical lesions may also show signs of venous congestion, their frequency in our TIA population was scarce compared to stroke populations [25].

In our small population positive image findings did not influence the post-TIA stroke risk, nor did anyone among the 22 patients with perfusion deficits only develop infarction signs at 8-week follow-up, perhaps due to small sample size.

In our clinical setting without external post-processing software the speed and accessibility of diffusion and ASL perfusion imaging enabled us to increase ischemia detection in comparison to diffusion imaging alone. This may aid in identification of high-risk patients with TIA with imaging abnormalities. Our standard vendor pulsed ASL sequence proved robust and identified perfusion changes larger than diffusion lesions and showed perfusion change in DWI-negative patients. ATA often bordered hypoperfused areas and served as an easily identifiable pointer useful in the clinical situation, and we deemed it worth the extra scan time.

\section{Conclusions}

Ischemia detection is improved by adding ASL to the TIA protocol, and the standard sequence proved feasible and robust in a clinical setting. SWI did not add critical information in subacute diagnostic work-up for TIA. Localization and underlying local vascularity seem to be key factors in the morphologic development of ischemia lesion depth and long-term traces.

\section{Additional files}

Additional file 1: Supplementary methodology. Motion artefact assessment. Supplemental results. Table S1. Combinations of number of planes and images affected by subject motion for $n=116$ patients. Table S2. Large vessel 
findings for patients ( $n=116$ ) stratified after most likely etiology after full radiological and clinical workup. Table S3. Risk of persistent infarction signs for DWI lesions and perfusion findings. (DOCX $20 \mathrm{~kb}$ )

Additional file 2: Figure S1. ASL interpretability and artefact description for 116 patients. Top 4 rows compare ASL PWI and relCBF images. Bottom row shows ring artefact frequency. $\mathrm{GW}=$ gray-white matter discrimination. (PDF 4 kb)

\section{Abbreviations}

ASL: Arterial spin labeling; ATA: Arterial transit artefact; CBF: Cerebral blood flow; cGM: Cortical gray matter; Cl: Confidence interval; CRF: Case report form; dGM: Deep gray matter; DSC: Dynamic susceptibility contrast; DW: Diffusion weighted imaging; FLAIR: Fluid attenuated inversion recovery; HR: Hazard ratio; IQR: Interquartile range; MRI: Magnetic resonance imaging; NIHSS: National Institutes of Health Stroke Scale; OR: Odds ratio; SWl: Susceptibility weighted imaging; TIA: Transient ischemic attack; TOAST: Trial of ORG 10172 in acute stroke treatment; WM: White matter

\section{Funding}

CO holds research grants from the Velux-foundation, Bispebjerg University Hospital, University of Copenhagen, Axel Muusfeldts Foundation and Danish Medical Association. None were designated for this study.

\section{Availability of data and materials}

The datasets generated and/or analyzed during the current study are available upon publication in the Figshare repository, DOI https://doi.org/10.6084/ m9.figshare.5331193.

\section{Authors' contributions}

$\mathrm{IH}, \mathrm{HC}, \mathrm{AC}$ conceived and designed the study. All authors were involved in data acquisition. KE, JM, PM, SR, MF, HC were involved in patient inclusion. LW performed the clinical follow-up and provided critical feed-back in the manuscript writing. $\mathrm{H}, \mathrm{CO}, \mathrm{JD}, \mathrm{AC}, \mathrm{HC}$ analyzed and interpreted data. $\mathrm{IH}$ wrote the first draft. All authors reviewed and edited the manuscript and approved the final version of the manuscript.

\section{Ethics approval and consent to participate}

The Danish National Committee of Biomedical Research Ethics (H-1-2011-75) approved the study, ClinicalTrials.gov Identifier NCT01531946 and it was performed according to the Declaration of Helsinki.

\section{Consent for publication}

Patients were included after written informed consent.

\section{Competing interests}

The authors declare that they have no competing interests.

\section{Publisher's Note}

Springer Nature remains neutral with regard to jurisdictional claims in published maps and institutional affiliations.

\section{Author details}

'Department of Radiology, Copenhagen University Hospital Bispebjerg, Bispebjerg Bakke 23, 2400 Copenhagen, NV, Denmark. ${ }^{2}$ Department of Neurology, Copenhagen University Hospital Bispebjerg, Bispebjerg Bakke 23, 2400 Copenhagen, NV, Denmark

Received: 30 August 2017 Accepted: 30 July 2018

Published online: 20 August 2018

\section{References}

1. Amarenco P, Lavallée PC, Labreuche J, Albers GW, Bornstein NM, Canhão P, et al. One-year risk of stroke after transient ischemic attack or minor stroke. N Engl J Med. 2016;374:1533-42.

2. Castle J, Mlynash M, Lee K, Caulfield AF, Wolford C, Kemp S, et al. Agreement regarding diagnosis of transient ischemic attack fairly low among stroketrained neurologists. Stroke. 2010;41:1367-70.

3. Zaharchuk G, Olivot J-M, Fischbein NJ, Bammer R, Straka M, Kleinman JT, et al. Arterial spin labeling imaging findings in transient ischemic attack patients: comparison with diffusion- and bolus perfusion-weighted imaging Cerebrovasc Dis. 2012;34:221-8.

4. Chen Y, Wang DJ, Detre JA. Test-retest reliability of arterial spin labeling with common labeling strategies. J Magn Reson Imaging. 2011:33:940-9.

5. Deibler AR, Pollock JM, Kraft RA, Tan H, Burdette JH, Maldjian JA. Arterial spin-labeling in routine clinical practice, part 1: technique and artifacts. AJNR Am J Neuroradiol. 2008;29:1228-34.

6. Viallon M, Altrichter S, Pereira VM, Nguyen D, Sekoranja L, Federspiel A, et al. Combined use of pulsed arterial spin-labeling and susceptibility-weighted imaging in stroke at 3T. Eur Neurol. 2010;64:286-96.

7. Hernandez DA, Bokkers RPH, Mirasol RV, Luby M, Henning EC, Merino JG, et al. Pseudo-continuous arterial spin labeling quantifies relative cerebral blood flow in acute stroke. Stroke. 2012:43:753-8.

8. Zaharchuk G, El Mogy IS, Fischbein NJ, Albers GW. Comparison of arterial spin labeling and bolus perfusion-weighted imaging for detecting mismatch in acute stroke. Stroke. 2012;43:1843-8.

9. Wang DJ, Alger JR, Qiao JX, Hao Q, Hou S, Fiaz R, et al. The value of arterial spin-labeled perfusion imaging in acute ischemic stroke comparison with dynamic susceptibility contrast enhanced MRI. Stroke. 2012;43:1018-24.

10. Maclntosh BJ, Lindsay AC, Kylintireas I, Kuker W, Günther M, Robson MD, et al. Multiple inflow pulsed arterial spin-labeling reveals delays in the arterial arrival time in minor stroke and transient ischemic attack. AJNR Am J Neuroradiol. 2010;31:1892-4

11. Asdaghi N, Hameed B, Saini M, Jeerakathil T, Emery D, Butcher K. Acute perfusion and diffusion abnormalities predict early new MRI lesions 1 week after minor stroke and transient ischemic attack. Stroke. 2011;42:2191-5.

12. Zaharchuk G, Bammer R, Straka M, Shankaranarayan A, Alsop DC, Fischbein $\mathrm{NJ}$, et al. Arterial spin-label imaging in patients with normal bolus perfusionweighted MR imaging findings: pilot identification of the Borderzone sign. Radiology. 2009;252:797-807

13. Deibler AR, Pollock JM, Kraft RA, Tan H, Burdette JH, Maldjian JA. Arteria spin-labeling in routine clinical practice, part 2: Hypoperfusion patterns. AJNR Am J Neuroradiol. 2008;29:1235-41.

14. Qiao XJ, Salamon N, Wang DJ, He R, Linetsky M, Ellingson BM, et al. Perfusion deficits detected by arterial spin labeling (ASL) in TIA patients with negative diffusion and vascular imaging. AJNR Am J Neuroradiol. 2013:34:2125-30.

15. Chalela JA, Alsop DC, Gonzalez-Atavales JB, Maldjian JA, Kasner SE, Detre JA. Magnetic resonance perfusion imaging in acute ischemic stroke using continuous arterial spin labeling. Stroke. 2000;31:680-7.

16. Wolf RL, Alsop DC, McGarvey ML, Maldjian JA, Wang J, Detre JA. Susceptibility contrast and arterial spin labeled perfusion MRI in cerebrovascular disease. J Neuroimaging. 2003;13:17-27.

17. Kleinman JT, Zaharchuk G, Mlynash M, Ogdie AA, Straka M, Lansberg MG, et al. Automated perfusion imaging for the evaluation of transient ischemic attack. Stroke. 2012:43:1556-60.

18. Mlynash M, Olivot J-M, Tong DC, Lansberg MG, Eyngorn I, Kemp S, et al. Yield of combined perfusion and diffusion MR imaging in hemispheric TIA. Neurology. 2009;72:1127-33.

19. Tong T, Yao Z, Feng X. Combined diffusion- and perfusion-weighted imaging: a new way for the assessment of hemispheric transient ischemic attack patients. Int J Dev Neurosci. 2011;29:63-9.

20. Nah H-W, Kwon SU, Kang D-W, Lee D-H, Kim JS. Diagnostic and prognostic value of multimodal MRI in transient ischemic attack. Int. J. Stroke off. J Int Stroke Soc. 2014;9:895-901.

21. Lee SH, Nah HW, Kim BJ, Ahn SH, Kim JS, Kang DW, et al. Role of perfusionweighted imaging in a diffusion-weighted-imaging-negative transient ischemic attack. J Clin Neurol. 2017;13:129-37.

22. Mittal S, Wu Z, Neelavalli J, Haacke EM. Susceptibility-weighted imaging: technical aspects and clinical applications, part 2. AJNR Am J Neuroradiol. 2009;30:232-52

23. Kesavadas C, Thomas B, Pendharakar H, Sylaja PN. Susceptibility weighted imaging: does it give information similar to perfusion weighted imaging in acute stroke? J Neurol. 2011;258:932-4.

24. Baik SK, Choi W, Oh SJ, Park K-P, Park M-G, Yang TI, et al. Change in cortical vessel signs on susceptibility-weighted images after full recanalization in Hyperacute ischemic stroke. Cerebrovasc Dis. 2012;34:206-12.

25. Jensen-Kondering $U$, Bohm R. Asymmetrically hypointense veins on $T 2^{*} W$ imaging and susceptibility-weighted imaging in ischemic stroke. World J Radiol. 2013;5:156-65. 
26. Kaya D, Dinçer A, Yildiz ME, Çizmeli MO, Erzen C. Acute ischemic infarction defined by a region of multiple Hypointense vessels on gradient-Echo $T 2^{*}$ MR imaging at 3T. AJNR Am J Neuroradiol. 2009;30:1227-32.

27. Qin L, van Gelderen P, Derbyshire JA, Jin F, Lee J, de Zwart JA, et al. Prospective head-movement correction for high-resolution MRI using an in-bore optical tracking system. Magn Reson Med. 2009;62:924-34.

28. Kober T, Gruetter R, Krueger G. Prospective and retrospective motion correction in diffusion magnetic resonance imaging of the human brain. Neurolmage. 2012;59:389-98.

29. Dold C, Zaitsev M, Speck O, Firle EA, Hennig J, Sakas G. Prospective head motion compensation for MRI by updating the gradients and radio frequency during data acquisition. Med Image Comput Comput Assist Interv. 2005;8:482-9.

30. Andre JB, Bresnahan BW, Mossa-Basha M, Hoff MN, Smith CP, Anzai Y, et al. Toward quantifying the prevalence, severity, and cost associated with patient motion during clinical MR examinations. J Am Coll Radiol. 2015; 12:689-95.

31. Detre JA, Samuels OB, Alsop DC, Gonzalez-At JB, Kasner SE, Raps EC. Noninvasive magnetic resonance imaging evaluation of cerebral blood flow with acetazolamide challenge in patients with cerebrovascular stenosis. J Magn Reson Imaging. 1999;10:870-5.

32. Asdaghi N, Campbell BCV, Butcher KS, Coulter Jl, Modi J, Qazi A, et al. DWI reversal is associated with small infarct volume in patients with TIA and Minor stroke. AJNR Am J Neuroradiol. 2014;35:660-6.

33. Merwick A, Albers GW, Amarenco P, Arsava EM, Ay H, Calvet D, et al. Addition of brain and carotid imaging to the $A B C D^{2}$ score to identify patients at early risk of stroke after transient ischaemic attack: a multicentre observational study. Lancet Neurol. 2010;9:1060-9.

34. Purroy F, Begué R, Quílez A, Piñol-Ripoll G, Sanahuja J, Brieva L, et al. The California, $A B C D$, and unified $A B C D 2$ risk scores and the presence of acute ischemic lesions on diffusion-weighted imaging in TIA patients. Stroke. 2009:40:2229-32.

35. Ay H, Arsava EM, Johnston SC, Vangel M, Schwamm LH, Furie KL, et al. Clinical- and imaging-based prediction of stroke risk after transient ischemic attack the CIP model. Stroke. 2009;40:181-6.

36. Giles MF, Albers GW, Amarenco P, Arsava EM, Asimos AW, Ay H, et al. Early stroke risk and ABCD2 score performance in tissue- vs time-defined TIA. Neurology. 2011;77:1222-8

37. Lavallée PC, Meseguer E, Abboud H, Cabrejo L, Olivot J-M, Simon O, et al. A transient ischaemic attack clinic with round-the-clock access (SOS-TIA): feasibility and effects. Lancet Neurol. 2007;6:953-60.

38. Zaitsev M, Maclaren J, Herbst M. Motion artifacts in MRI: a complex problem with many partial solutions. J Magn Reson Imaging. 2015;42:887-901.

39. Cho HJ, Yang JH, Jung YH, Kim YD, Choi H-Y, Nam HS, et al. Cortex-sparing infarctions in patients with occlusion of the middle cerebral artery. J Neurol Neurosurg Psychiatry. 2010;81:859-63.

40. Brozici M, van der Zwan A, Hillen B. Anatomy and functionality of Leptomeningeal anastomoses a review. Stroke. 2003;34:2750-62.

41. Schwamm LH, Koroshetz WJ, Sorensen AG, Wang B, Copen WA, Budzik R, et al. Time course of lesion development in patients with acute stroke serial diffusion- and hemodynamic-weighted magnetic resonance imaging. Stroke. 1998;29:2268-76.

42. van Gelderen P, de Zwart JA, Duyn JH. Pittfalls of MRI measurement of white matter perfusion based on arterial spin labeling. Magn Reson Med. 2008:59:788-95.

43. van Osch MJ, Teeuwisse WM, van Walderveen MAA, Hendrikse J, Kies DA, van Buchem MA. Can arterial spin labeling detect white matter perfusion signal? Magn Reson Med. 2009;62:165-73.

Ready to submit your research? Choose BMC and benefit from:

- fast, convenient online submission

- thorough peer review by experienced researchers in your field

- rapid publication on acceptance

- support for research data, including large and complex data types

- gold Open Access which fosters wider collaboration and increased citations

- maximum visibility for your research: over $100 \mathrm{M}$ website views per year

At BMC, research is always in progress.

Learn more biomedcentral.com/submissions 